\title{
CINTED-UFRGS
OBJETOS DE APRENDIZAGEM NO ENSINO DE TÓPICOS DE ELETRODINÂMICA
}

\section{LEARNING OBJECTS IN ELECTRODYNAMICS TOPICS TEACHING}

\author{
Anderson Luis Ellwanger - Centro Universitário Franciscano, pfandd@gmail.com \\ Jussane Rossato - Centro Universitário Franciscano, jussaner@gmail.com \\ Valeria Iensen Bortoluzzi - Centro Universitário Franciscano, \\ valeria.bortoluzzi@gmail.com \\ Solange Binotto Fagan - Centro Universitário Franciscano, solange.fagan@gmail.com
}

\section{RESUMO}

Neste artigo, abordamos tópicos de eletrodinâmica via objetos virtuais de aprendizagem. O material didático foi desenvolvido por uma equipe pedagógica responsável pela elaboração dos conteúdos em roteiros, e por uma equipe técnica, responsável por transformar os roteiros dos conteúdos em produtos tecnológicos. O recurso didático foi desenvolvido no Centro Universitário Franciscano, na cidade de Santa Maria, Rio Grande do Sul - Brasil. Os objetos e os conteúdos sobre eletrodinâmica estão alocados num espaço virtual de aprendizagem denominado MAIS Unifra e foram testados por estudantes de diferentes cursos de graduação e avaliados por professores especialistas. Os conteúdos abordados estão relacionados com conceitos de eletrodinâmica e contemplam demonstrações computacionais, animações, vídeos e hipertextos. Ao final dos conteúdos, há atividades que podem ser desenvolvidas pelos usuários e também sugestões para a sua aplicação em sala de aula presencial ou à distância.

Palavras-chave: eletrodinâmica, objetos de aprendizagem, ensino.

\begin{abstract}
In this paper, we present electrodynamics topics via virtual learning objects. The courseware has been developed by an educational team responsible for planning the content of roadmaps, and a technical team responsible for transforming the contents of the scripts in technological products. The teaching resource was developed at the Franciscan University Center, in Santa Maria, Rio Grande do Sul - Brazil. The electrodynamics object is allocated in a virtual learning space called MAIS UNIFRA, and tested by students of distinct undergraduate courses and assessed by specialist teachers. The subjects covered are related to concepts of computational electrodynamics and include demonstrations, animations, videos and hypertext. It is also present activities that can be developed by the users and also suggestions for use in the classroom or e-learning.
\end{abstract}

Keywords: electrodynamics, learning objects, education. 


\section{INTRODUÇÃO}

O ensino de Física tem enfrentado diversos desafios, dentre eles podemos destacar a falta de professores qualificados, currículos engessados, carga horária insuficiente para a abordagem adequada dos conceitos. Além disso, soma-se a desatualização das práticas pedagógicas, amparadas quase que exclusivamente em métodos pouco interativos. É possível perceber, assim, que o ensino de Física na Educação Básica está em crise, aumentando as chances de gerar uma aprendizagem transmissiva ou mecânica e pouco significativa (MOREIRA, 2011).

Para contornar o panorama atual, algumas tentativas vêm sendo apresentadas, entre elas temos a abordagem interdisciplinar, o uso de novas tecnologias, a aprendizagem pela pesquisa, entre outras. Fagan e colaboradores (2011) desenvolveram objetos virtuais de aprendizagem relacionados com o "custo do banho" e o "código de cores", nos quais verificaram que os discentes demonstraram maior interesse e entendimento sobre os conceitos abordados. Ellwanger e colaboradores (2012) abordaram tópicos relacionados com a Nanociência, por meio de objetos virtuais de aprendizagem, e observaram que estes objetos e conteúdos facilitam a compreensão de conceitos que são considerados complexos e abstratos e envolvem uma escala muito pequena, que é o nanômetro.

As alternativas pedagógicas que fazem uso de recursos digitais existentes podem apresentar conteúdos relacionados com Física Clássica de forma diferenciada aos estudantes e, assim, a aprendizagem, por parte deles, tornar-se-á mais atraente e talvez menos mecânica.

Portanto, neste artigo, exploramos o uso de novas tecnologias no ensino, vislumbrando um enfoque diferenciado para abordar tópicos de Física Clássica, especificamente Eletrodinâmica, por meio de objetos virtuais de aprendizagem. O estudo de áreas vinculadas com circuitos elétricos é um dos ramos da Física mais abrangentes, visto que são incorporados em todos os componentes eletrônicos. Nesta perspectiva, a compreensão do mundo que nos cerca passa obrigatoriamente pela compreensão dos fenômenos elétricos (DORNELES; ARAUJO; VEIT, 2008).

Assim sendo, nosso objetivo com esta proposta é apresentar um material virtual, potencialmente significativo, que foi desenvolvido para o ensino de tópicos de Eletrodinâmica. $\mathrm{O}$ desenvolvimento do conteúdo digital amparou-se na familiaridade dos usuários com as tecnologias e também nos pressupostos da aprendizagem significativa (MOREIRA, 2005) e nos campos conceituais de Vergnaud (1993).

$\mathrm{Na}$ próxima seção, enfatizamos o referencial teórico usado no desenvolvimento deste trabalho.

\section{REFERENCIAL TEÓRICO}

Dividimos o referencial teórico empregado neste estudo em referencial de aprendizagem e referencial de trabalho, os quais serão descritos detalhadamente nas próximas subseções. Inicialmente, iremos destacar o referencial de aprendizagem. 


\subsection{Referencial de Aprendizagem}

O estudo da Eletrodinâmica pode ser dividido em duas frentes. Na primeira, cientistas e técnicos usam a abordagem matemática e suas ferramentas para prever a construção de novos circuitos e dispositivos eletrônicos. Neste contexto, são necessários conhecimentos específicos, ignorados pela maioria da população. Numa segunda perspectiva, ocorre a abordagem conceitual, possível de ser compartilhada por um grupo maior de pessoas, inclusive estudantes e comunidade em geral.

Visando o enfoque conceitual e assumindo uma postura pautada na possibilidade de incentivar a busca por conhecimentos científicos, julgamos adequado embasar essa produção didática na teoria da aprendizagem significativa (MOREIRA, 2005) e na teoria psicológica da conceitualização do real (VERGNAUD, 1993).

$\mathrm{Na}$ perspectiva da aprendizagem significativa (AUSUBEL, 2003), os novos conhecimentos passam a fazer parte da vida do indivíduo, de forma ampla e relacionada com os demais conhecimentos que ele já possuía. Esses aspectos são os fatores mais relevantes para a aprendizagem de um novo assunto. Desta forma, a instrução de conteúdos que possuam relação com o cotidiano dos estudantes pode gerar condições favoráveis a uma aprendizagem (MOREIRA, 2005).

O desenvolvimento de tópicos relacionados com Eletrodinâmica via objetos virtuais de aprendizagem, permitirá ao usuário manusear as ferramentas pelo tempo que julgar necessário para compreender os conceitos relacionados ao tópico. Poderá ainda manusear o aplicativo inúmeras vezes, aumentando e aprofundando sua compreensão conceitual.

Esta perspectiva colabora com os encaminhamentos de Vergnaud (1993), que designa ser a abstração conceitual o mais relevante no processo. Neste processo de conceitualização do real, o indivíduo assimila novos conhecimentos, associando-os aos seus esquemas conceituais existentes e, em muitos casos, reestruturando-os e aprimorando-os (VERGNAUD, 1993).

A conceitualização do real consiste em assimilar a realidade, por meio de esquemas conceituais existentes na episteme do indivíduo, de forma a situar e relacionar as dependências e independências entre determinados conceitos, em especial com referência às aprendizagens científicas e técnicas (VERGNAUD, 1993). Os conceitos são assimilados ao longo do tempo, o que promove a modificação e o aprimoramento do indivíduo que os absorve, transforma, amplia e melhora a sua compreensão, e, em muitos casos, substitui conceitos anteriores.

Portanto, consideramos que a exploração de tópicos de Eletrodinâmica, usando aplicativos virtuais não lineares e não dependentes do tempo, embasados teoricamente na abordagem conceitual, pode gerar condições favoráveis para a aprendizagem significativa.

\subsection{Referencial de trabalho}

A transposição didática entre a produção científica e tecnológica e os ambientes de ensino é incentivada por norteadores curriculares como os PCN, visando a uma instrução mais atualizada e contextual (BRASIL, 2002). O processo através do qual o "saber sábio", aquele que é produzido pelos cientistas, transforma-se em "saber a ensinar", aquele que está contido nos programas e livros didáticos e, consequentemente, aquele que efetivamente vai para a sala de aula, o "saber ensinado", acaba gerando modificações nos tópicos transpostos, que mantêm algumas características iniciais, porém adquirem significados 
próprios no contexto escolar e um novo status epistemológico (ASTOLFI; DEVALAY, 1995).

O processo de transposição didática (CHEVALLARD, 2005) deve obedecer a cinco regras, conforme destacam Brockmington e Pietrocola (2005):

Regra 1 - Modernizar o saber escolar;

Regra 2 - Atualizar a metodologia do saber a ensinar;

Regra 3 - Articular saber velho com saber novo;

Regra 4 - Transformar um saber em exercícios e problemas investigados;

Regra 5 - Tornar um conceito mais compreensível.

Essas regras, adequadas ao contexto do presente estudo, podem ser expostas da seguinte forma:

Regra 1- A modernização do saber escolar pode ser alcançada por meio da inserção de conceitos de eletrodinâmica nos ambientes virtuais de ensino.

Regra 2- A atualização da metodologia do saber a ensinar foi desenvolvida usando uma abordagem didática adequada aos PCN.

Regra 3- A relação entre saberes velho e novo será articulada por meio dos conceitos da Física Clássica, como força, campo e potencial, e seus desdobramentos em diferentes escalas de medida e na inserção de aplicações do dia a dia do estudante.

Regra 4 - Os efeitos observados de forma clássica e macroscópica e seus impactos em conceitos como resistência e armazenamento de dados, e que podem ser vistos como uma possibilidade de transformar um saber em exercícios e problemas.

Regra 5 - A compreensão de tópicos de eletrodinâmica de uma forma menos abstrata. Essas diretrizes foram norteadoras deste trabalho e estão amparadas também no referencial teórico de aprendizagem.

\section{OBJETOS DE APREDIZAGEM}

O Centro Universitário Franciscano conta, atualmente, com um repositório virtual em permanente transformação, denominado MAIS Unifra (MAIS UNIFRA, 2014). Neste espaço há conteúdos digitais e objetos de aprendizagem de autoria de uma equipe de professores, estudantes de graduação e de pós-graduação da instituição. A equipe também conta com o auxílio de profissionais que transformam os roteiros dos conteúdos digitais em produtos tecnológicos.

O traço definidor dos conteúdos e objetos existentes no MAIS Unifra é a interatividade, a qual se faz presente por meio de hipertextos e links direcionais. Já o diferencial do MAIS Unifra está nos fóruns moderados e chats interativos, onde os usuários podem interagir com outros usuários e encaminhar sugestões sobre os conteúdos e o ambiente à equipe responsável. Sendo assim, o MAIS Unifra (MAIS UNIFRA, 2014) caracteriza-se como um ambiente de aprendizagem concretizado de forma compartilhada e em rede.

Dentre os conteúdos desenvolvidos pela equipe pedagógica e técnica, daremos ênfase, neste artigo, aos conteúdos didáticos "Resistores e Lei de Ohm". Estes temas foram abordados com o objetivo de introduzir tópicos indispensáveis para a compreensão inicial de conceitos relacionados com Eletrodinâmica.

Nas subseções 3.1 e 3.2, faremos a descrição dos objetos de aprendizagem apresentados nos conteúdos digitais "Resistores" e "Lei de Ohm" disponíveis no espaço virtual de aprendizagem MAIS Unifra (MAIS UNIFRA, 2014). 


\subsection{Resistores}

Os objetivos de aprendizagem deste conteúdo foram: identificar a resistência nominal de diferentes resistores, usando o código de cores; analisar os resistores em um circuito elétrico; selecionar resistores de um conjunto, de modo a projetar circuitos elétricos.

Para que o usuário possa identificar o valor de um resistor, foi organizado um conteúdo explicativo bem como um objeto de aprendizagem. Este objeto interativo auxilia o usuário a identificar os valores de um resistor por meio do código de cores. Nesta atividade, o usuário obtém informações sobre o significado das listas coloridas dos resistores, além de construir resistores usando a tabela com o código de cores. Na Figura 1, temos a representação esquemática do objeto "Como identificar o valor de um resistor".

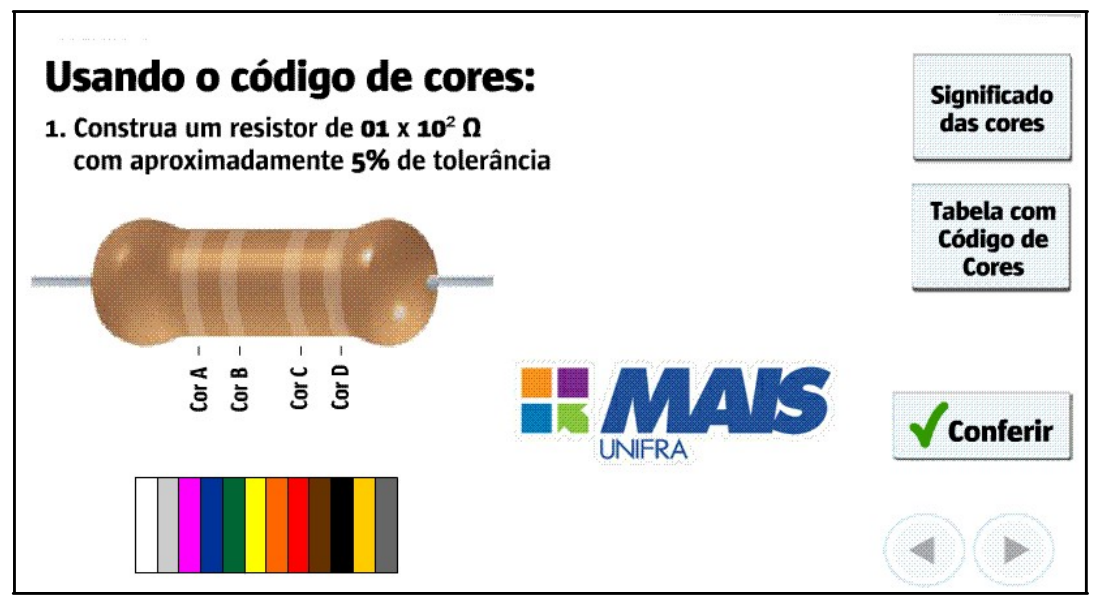

Figura 1 - Representação esquemática da página inicial do objeto de aprendizagem “Como identificar o valor de um resistor”. Objeto disponível em: http://maisunifra.com.br/conteudo/resistores/\#6.

\subsection{Lei de Ohm}

Este conteúdo didático tem como objetivos de aprendizagem: definir e usar a Lei de Ohm para os elementos resistivos ôhmicos; diferenciar elementos resistivos ôhmicos de não ôhmicos; distinguir função quadrática de função linear, com base nos conceitos de condutor ôhmico e não ôhmico.

Na primeira atividade do objeto didático, "Gráfico da Lei de Ohm”, o usuário busca informações sobre a característica do resistor ôhmico por meio do gráfico animado que ilustra a lei de Ohm, a qual é representada por uma função linear em que a resistência permanece constante. No objeto denominado "Gráficos que obedecem a lei de Ohm", o usuário testa os conhecimentos sobre o conteúdo, escolhendo entre os diversos gráficos qual deles representa a lei de Ohm. Além desses objetos, ainda foi organizado um objeto denominado "Resistência do Circuito", no qual o usuário testa os conhecimentos sobre a Lei de Ohm e encontra a resistência resultante do circuito.

Ohm".

Na Figura 2, temos a representação esquemática do objeto "Gráfico da Lei de 


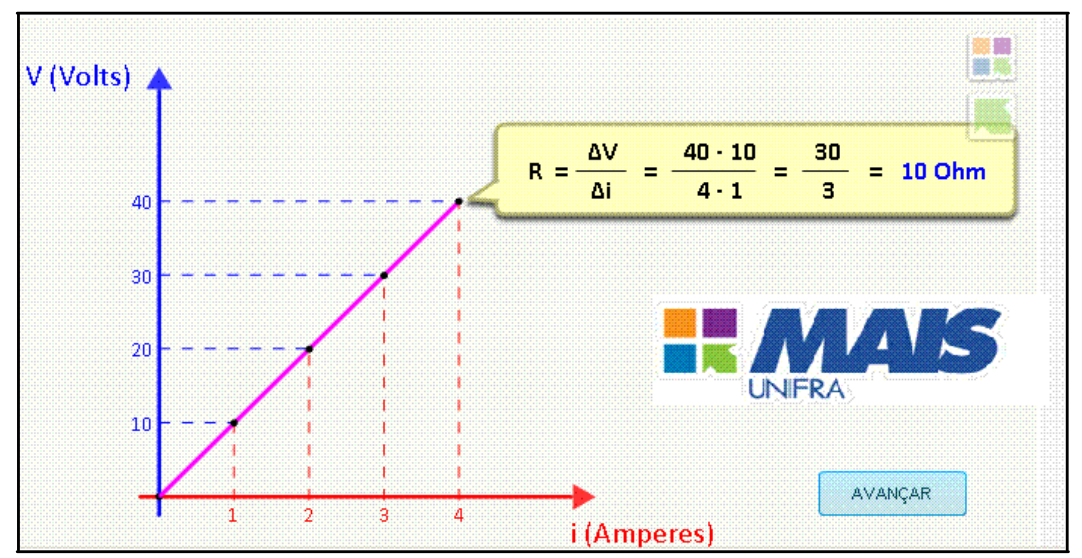

Figura 2 - Representação esquemática da página inicial do objeto de aprendizagem "gráfico da lei de Ohm". Objeto disponível em: http://maisunifra.com.br/conteudo/lei-de-ohm/\#3.

Na Figura 3, temos a representação esquemática do objeto "Gráficos que obedecem a Lei de Ohm".

Com os conhecimentos que você possui sobre a Lei de Ohm, escolha qual dos gráficos abaixo obedece a Lei de Ohm

A)

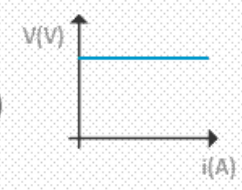

D)

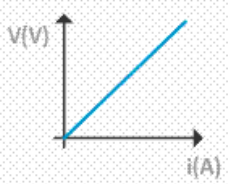

B)

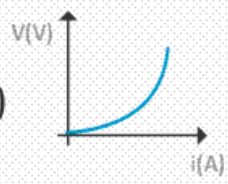

E)

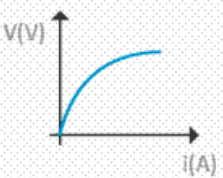

C)

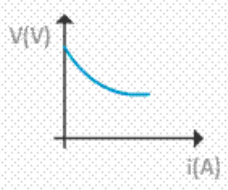

HMals

Figura 3 - Representação esquemática da página inicial do objeto de aprendizagem "gráficos que obedecem a lei de Ohm". Objeto disponível em: http://maisunifra.com.br/conteudo/lei-de-ohm/\#5.

Na Figura 4, temos a representação esquemática do objeto "Resistência do circuito".

Estes conteúdos, juntamente com os objetos de aprendizagem, desenvolvidos na unidade didática Eletrodinâmica, permanecerão em constante atualização, para que, sempre que possível, se adequem às sugestões dos usuários. As sugestões e arguições, por meio de chats, fóruns e e-mail, mantêm a equipe pedagógica em constante vigilância epistemológica e técnica.

Nesta perspectiva buscamos, nos espaços de diálogo, sempre que possível, indícios de aprendizagem significativa por parte dos usuários. Além disso, discutimos e repensamos a estruturação dos objetos e conteúdos, buscando abordagens mais completas e acessíveis aos seus usuários. 

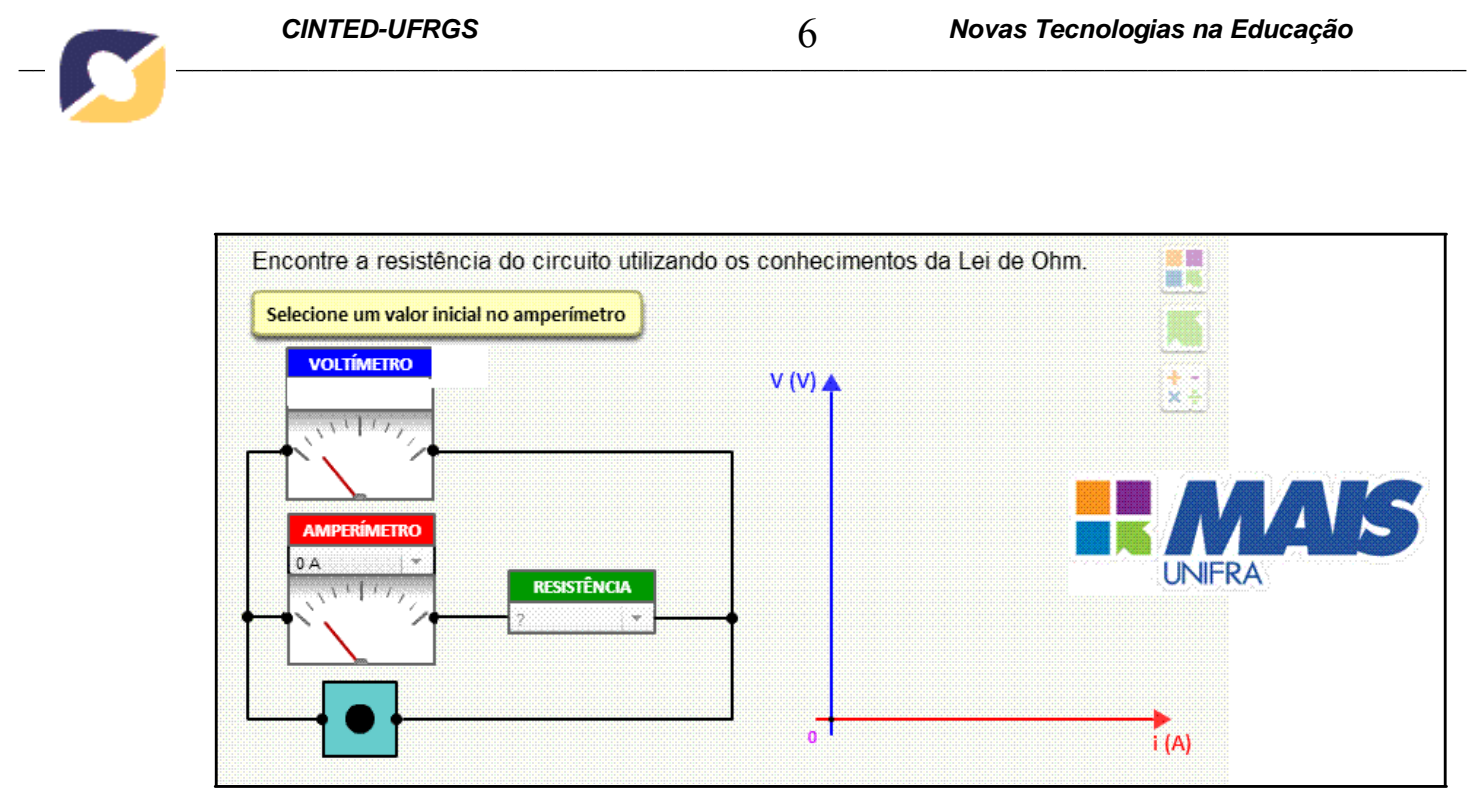

Figura 4 - Representação esquemática da página inicial do objeto de aprendizagem "resistência do circuito". Objeto disponível em: http://maisunifra.com.br/conteudo/lei-de-ohm/\#4.

Para finalizar, apresentamos o resultado da avaliação dos objetos de aprendizagem por parte de estudantes e professores especialistas da área.

\section{3. Avaliações dos objetos de aprendizagem}

Os objetos de aprendizagem, explanados neste artigo, foram testados por acadêmicos do curso de Física Médica do Centro Universitário Franciscano, Santa Maria RS, e também de outras áreas. Os acadêmicos usuários apresentaram sugestões e identificaram os aspectos que julgavam pouco precisos. Durante a avaliação, que foi registrada em vídeo, os graduandos observaram a recursividade e a não linearidade dos aplicativos como um dos pontos mais relevantes do material, o que, segundo eles, aumenta as chances de compreensão do assunto.

Após esta etapa, professores especialistas foram convidados a avaliar os objetos. Estes ratificaram a consistência teórica e julgaram os materiais úteis para a sua prática. As solicitações relevantes, tanto dos acadêmicos bem como dos professores, foram contempladas na reestruturação dos objetos.

Atualmente, os objetos de aprendizagem constituem uma ferramenta auxiliar na prática pedagógica, sendo utilizados por professores de diferentes cursos de graduação e de pós-graduação do Centro Universitário Franciscano para abordagem do tema Eletrodinâmica.

\section{CONCLUSÕES}

Neste trabalho, apresentamos a elaboração, a avaliação e a publicação de conteúdos digitais para ambientes virtuais de aprendizagem relacionados com a Eletrodinâmica. Os materiais digitais produzidos permitem a complementação das atividades desenvolvidas em sala de aula, presencial ou virtual, tornando, desta forma, o ensino mais atrativo e interligado com o cotidiano dos estudantes. Esses materiais podem ser acessados e explorados de forma independente ou associados a outros tópicos disponíveis no mesmo ambiente virtual. A interação entre usuários e a equipe pedagógica ocorre por meio de chats e fóruns de discussões, permitindo desdobramentos e esclarecimento de dúvidas.

Além disso, ressaltamos que a transposição didática foi norteadora na produção dos objetos de aprendizagem, na organização dos hipertextos, buscando facilitar a assimilação dos conceitos, abordados em Eletrodinâmica, para o usuário. Portanto, a produção de 
objetos de aprendizagem que contemplem tecnologias atuais, aliadas a novas metodologias de ensino, poderão auxiliar professores e estudantes de diferentes níveis de ensino em suas práticas educacionais, tornando o ensino mais atualizado e atrativo. Esta metodologia busca uma maior interatividade entre os usuários e também incentiva o conhecimento acerca de temas atuais.

\section{REFERÊNCIAS BIBLIOGRÁFICAS}

ASTOLFI, J. P; DEVELAY, M. A Didática das Ciências. 4. ed. Campinas: Papirus, 1995. $132 \mathrm{p}$.

AUSUBEL, D. P. Aquisição e retenção de conhecimentos: uma perspectiva cognitiva. 1. ed. Lisboa: Plátano, 2003. 219 p.

BRASIL. Ministério da Educação, Secretaria de Educação Média e Tecnológica. Parâmetros curriculares nacionais: Ensino Médio. Brasília, MEC/SEMT, 2002.

BROCKINGTON, G.; PIETROCOLA, M. Serão as regras da Transposição Didáticas aplicáveis aos conceitos de Física Moderna? Investigações em Ensino de Ciências, Porto Alegre, v. 10, n. 3, p. 387-404, 2005.

CHEVALLARD, Y. La Transposición Didáctica: del saber sabio al saber enseñado. 3. ed. Buenos Aires: Aique, 2005. 196 p.

DORNELES, P., ARAUJO, I. S.; VEIT, E.A. Simulação e modelagem computacionais no auxílio da aprendizagem significativa de conceitos básicos de eletricidade. Parte II circuitos RLC. Revista Brasileira de Ensino de Física, v. 30, n. 3, p. 3308-1 - 3308-16, 2008.

ELLWANGER, A. L.; ROSSATO, J. ; GRANADA, M.; BORTOLUZZI, V. I; FAGAN, S. B. O Ensino de Nanociências por meio de Objetos de Aprendizagem. Renote. Revista Novas Tecnologias na Educação, v. 10, p. 1-10, 2012.

FAGAN, S. B.; PORTO, A. V. L.; JAURIS, I. Objetos de aprendizagem para o ensino de Física: Custo do Banho e Código de Cores. Disciplinarum Scientia. Série: Ciências Naturais e Tecnológicas, v. 12, n. 1, p. 159-170, 2011.

MAIS UNIFRA. Espaço Virtual de Aprendizagem. Santa Maria, RS: Unifra, 2014. Disponível em: <http://maisunifra.com.br/>. Acesso em: 01de mai. 2014.

MOREIRA, M. A. Aprendizagem Significativa Crítica. Porto Alegre: Instituto de Física da UFRGS, 2005. 24 p.

MOREIRA, M. A. Teorias de Aprendizagem. São Paulo: EPU, 2011. 195 p.

VERGNAUD, G. Teoria dos Campos Conceituais, In Nasser, L. (Ed.) Anais do $1^{\circ}$ Seminário Internacional de Educação Matemática do Rio de Janeiro, p. 1-26, 1993. 